\title{
Numerical Investigation of Dimensionless Numbers on Macro-scale Synthetic Jet Actuator in Quiescent Flow
}

\author{
Mukhtar Ahmad, Adnan Qayoum* \\ Department of Mechanical Engineering, National Institute of Technology Srinagar, J\&K, 190006, India
}

Corresponding Author Email: adnan@nitsri.ac.in

https://doi.org/10.18280/ti-ijes.630108

Received: 26 January 2019

Accepted: 28 March 2019

\section{Keywords:}

synthetic jet Actuator, strokes length $(L)$, stokes number (S), active flow control, lift enhancement

\begin{abstract}
Synthetic jet is presently a novel technique for active flow control used for lift enhancement, improve boundary layer separation, and drag reduction, convective heat transfer enhancement and mixing enhancements. In this paper numerical simulation of macro-scale synthetic jet is carried out to investigate the effects of dimensionless numbers such as Stroke length (L), Stokes number $(\mathrm{S})$ and Reynolds numbers $\left(\mathrm{Re}_{\mathrm{L}}\right)$ at constant orifice diameter of $5 \mathrm{~mm}$. The computation is carried out by using commercial COMSOL Multiphysics 5.3a software for resolving the basic three dimensional incompressible unsteady Reynolds-averaged Navier-Stokes equations using k- $\varepsilon$ turbulence model coupled with Moving Mesh ALE module to address the oscillating diaphragm movement. The oscillating diaphragm attached to the bottom of cavity is modelled as movable wall boundary in a sinusoidal manner with a peak-to peak displacement is equal to $0.247 \mathrm{~mm}$ at constant resonant frequency of $10 \mathrm{~Hz}$. While the dimensionless Stroke length (L) ranges from 2 to 6, Stokes number $S=7.2$ to 22.9 and the Reynolds number establish on Stroke length $\operatorname{Re}_{\mathrm{L}}=11$ to 1080 at distinct orifice diameters. Grid independence study has been invoked to confirm the authentication of extracted computational results. The radial velocity profiles at the orifice exit obtained in CFD simulations are compared with existing experimental data. A reasonable agreement between the modelling and experiment has been observed in specific domains of the flow field. The result obtained in the present study provides the basic design code for synthetic jet flow parameters for optimum output.
\end{abstract}

\section{INTRODUCTION}

Flow control analysis investigates techniques and strategies capable of eliminating deteriorated aircraft performance characteristics at off-design conditions by unnaturally ever-changing and biasing the properties of the mean flow or its dynamics through the introduction of appropriate disturbances into the flow. Due to the necessity on transform aviation linked with the physical environment and cost compulsion ongoing to present changes in the aircraft architecture that will diminish acoustic loads, total vehicle drags and hazardous emissions while elevating structure safety, transport volume, range and endurance limit. The attention in synthetic jets actuators is mainly due to their usefulness in novel technique of flow control such as separation control and mixing enhancement. Glezer et al. [1] carried out experimental investigation that determines the criteria for the formation and evolution of synthetic jets in quiescent flow. Holman et al. [2] investigated the evolution and formation of synthetic jet which accounts generation and subsequent convection of vortex rings by time-averaged outward velocity along the axial direction. Jabbal et al. [3] carried out numerical simulation of synthetic jet actuator. It was revealed that roll-up was primarily characterised by the Stokes number. Smith et al. [4] studied characterization of synthetic jet actuator. They found the Reynolds number based on characteristics velocity of synthetic jet over the ejection phase with the conventional jets. Qayoum et al. [5] investigated the formation of synthetic jet using amplitude modulation. It was found that significant change was observed in the basic characteristics of synthetic jet. Zhang and Zhong [6] studied array of synthetic jet actuators by using induced florescence technique for laminar flow separation over a flap. The vortex structures produced by the interaction of cross flow for separation delay. Watson et al. [7] studied the least spacing between the multiple synthetic jets that allows formation and evolution of individual synthetic jets. McGuinn et al. [8] proposed experimental examination of circular synthetic jets using PIV and hot wire anemometry techniques. It was found that when the stroke length $(\mathrm{Lo} / \mathrm{D}=4)$ the strength of vortex ring increases forming the lagging behind trailing jet which weaken the vortex ring enhance mixing. Glezer and Amitay [9] reported a detailed review of flow characterization of synthetic jets in quiescent environment. You and Moin [10] studied synthetic jet impinging at different angle of attack and Reynolds number over a NACA0015 using Large-eddy simulations (LES). It was found that flow separation flow delays effectively thus increase lift coefficient. Jain et al. [11] numerically studied the influence of the cavity shape and orifice geometries of axisymmetric synthetic jet fluidics. They found that the efficiency of synthetic jets is more dependent on the size of orifice than that of the cavity. Lv et al. [12] studied the influence of the excitation frequency of synthetic jet actuator using SST k-w turbulence model. It was found that the synthetic jet fluidics are strongly depends on the thickness 
and radius of orifice. The ejection and suction capacity are restricted at lower orifice radius thus decreasing performance of synthetic jets. Batikh et al. [13] carried out experimental and numerical investigation for micro synthetic jet for flow control. The performance of synthetic jet actuator was modified by the augmentation of actuator frequency or by optimizing geometry of actuator and orifice.

The summary of the above literature demonstrates the utility of synthetic jets actuators for various active flow control utilizations such as drag declinement, lift augmentation, heat transfer impingement without study the dimensionless flow parameters associated with optimum construction of synthetic jet actuators. To this end, it is necessary to determine the impact of important dimensionless numbers associated on the characterization of synthetic jet actuators in order to maximize the orifice exit velocity that leads to better performance. The present study focuses on influence of dimensionless numbers on synthetic jet characterization such as optimum Stokes number (S), Stroke length (L) and Reynolds number depend on dimensionless Stroke length $\left(\mathrm{Re}_{\mathrm{L}}\right)$ at different orifice diameters. A schematic of a synthetic jet actuator is depicted in Figure 1 consists of a cylindrical cavity with an oscillatory diaphragm on its lower side and a circular orifice on the top of cavity which included necessary computational domain boundary conditions. The predominant geometric parameters of the synthetic jet actuator which involve diameter $\mathrm{D}_{\mathrm{o}}=5 \mathrm{~mm}$ and $0.5 \mathrm{~mm}$ and thickness $h_{0}=5 \mathrm{~mm}$ of the orifice, as well as diameter $D_{c}=45 \mathrm{~mm}$ and depth $h_{c}=10 \mathrm{~mm}$ of the cavity. The axial dimensions of surrounding quiescent air $\mathrm{B} 1=150 \mathrm{~mm}$ and that of lateral dimensions is $\mathrm{B} 2=100 \mathrm{~mm}$ respectively.

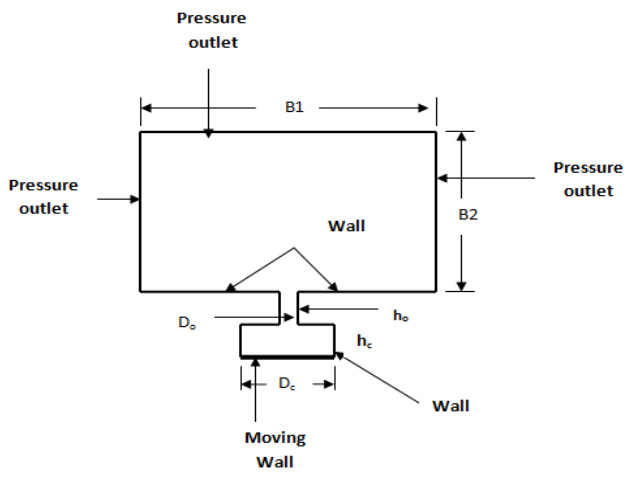

Figure 1. Schematic of a synthetic jet actuator showing boundary conditions at different walls and geometry of the computational domain

\section{COMPUTATIONAL DETAILS}

The numerical computation is performed by utilizing the Commercial CFD software COMSOL Multiphysics 5.3a based on finite element formulation for resolving three dimensional incompressible unsteady Reynolds-averaged Navier-Stokes equations using $\mathrm{k}-\boldsymbol{\varepsilon}$ turbulence model coupled with structural mechanics and ALE Moving Mesh module describing the oscillating sinusoidal motion of diaphragm. The present actuator diaphragm consists of a thin copper sheet. For discretization of different domains Lagrange quadratic rectangular elements scheme is used. The equations resolved are conservation of mass and momentum for the given geometry of computational domain. The present computational domain composed of three sections such as the cylindrical cavity, the circular orifice, and the surrounding ambient domain into which the synthetic jet exits. Holman et al. [2] demonstrated the criteria of synthetic jet formation based on Strouhal number is given by

$\frac{1}{\mathrm{~S}_{\mathrm{r}}}>C$

where $\mathrm{C}$ is the function of geometry of synthetic jet actuator and is equal to 0.06 . The sinusoidal vibrating motion of diaphragm consists of piston type profile whose instantaneous velocity can be expressed as

$u_{t}=\pi \Delta f \sin (2 \pi f t)$

where $f$ is the given excitation or actuation frequency of oscillating diaphragm of synthetic jet and $\Delta$ is given peak to peak displacement at the neutral position of diaphragm. The characteristics velocity (average velocity based over half ejection cycle) of synthetic jet is given by

$\mathrm{U}_{\mathrm{o}}=\mathrm{L} f=\int_{0}^{\mathrm{T} / 2} \mathrm{u}_{\mathrm{t}} \mathrm{dt}$

where $\mathbf{L}$ is the dimensionless Stroke length (distance that a slug travels during the ejection portion of the oscillation, $\mathrm{T}$ is the time period of actuation. In this study, air is taken as incompressible with constant physical properties.

The Reynolds Number based on average jet velocity at the orifice exit during the half of expulsion stroke is given by

$\operatorname{Re}_{U_{\mathrm{o}}}=\frac{\rho \mathrm{U}_{\mathrm{o}} \mathrm{D}_{\mathrm{o}}}{\mu}$

The governing equation of incompressible fluid inside the cylindrical cavity, the circular orifice and the surrounding medium is modeled by the incompressible Reynolds Averaged Navier- Stokes (RANS) equations as

Continuity Equation:

$\rho \nabla \cdot(\mathbf{u})=0$

Momentum equation:

$\rho(\mathbf{u} . \nabla) \mathbf{u}=\nabla \cdot\left[-\mathrm{P} \mathbf{I}+\left(\mu+\mu_{\mathrm{T}}\right) \nabla \mathbf{u}+(\nabla \mathbf{u})^{\mathrm{T}}-\frac{2}{3} \rho \mathrm{kI}+\mathbf{F}\right]$

where,

$\mu_{\mathrm{T}}=\rho \mathrm{C}_{\mu} \mathrm{k}^{2} / \varepsilon$

Also, k- $\varepsilon$ model adds two supplementry transport equations these includes the turbulent kinetic energy (k), and the dissipation rate of turbulence energy $(\varepsilon)$,

Turbulent kinetic energy equation $(k)$ :

$\rho(\mathbf{u} . \nabla) k=\nabla \cdot\left[\left(\mu+\frac{\mu_{T}}{\sigma_{k}}\right) \nabla k\right]+p_{k}-\rho \varepsilon$

Dissipation equation $(\varepsilon)$ :

$\rho(\mathbf{u} . \nabla) \varepsilon=\nabla \cdot\left[\left(\mu+\frac{\mu_{\mathrm{T}}}{\sigma_{\mathrm{s}}}\right) \nabla \varepsilon\right]+\mathrm{Ce}_{1} \frac{\varepsilon}{\mathrm{k}} \mathrm{p}_{\mathrm{k}}-\mathrm{Ce}_{2} \rho \varepsilon^{2} / \mathrm{k}$

where,

$\mathrm{p}_{\mathrm{k}}=\mu_{\mathrm{T}}\left[\nabla \mathbf{u}:\left(\nabla \mathbf{u}+\nabla \mathbf{u}^{\mathrm{T}}\right)\right]+\frac{2}{3} \rho \mathrm{k} \nabla \cdot \mathbf{u}$

$\mathrm{C}_{\mu}=0.09, \sigma_{\mathrm{k}}=1, \mathrm{Ce}_{1}=1.44$ and $\mathrm{Ce}_{2}=1.92$ 
The material properties of working fluid and input parameters for membrane in the governing equations are

$\rho=1.225 \mathrm{~kg} / \mathrm{m}^{3}, \mu=1.825 \times 10^{-5} \mathrm{~kg} / \mathrm{ms}$ and

$v=1.516 \times 10^{-5} \mathrm{~m}^{2} / \mathrm{s}$

Reynolds Number established on dimensionless stroke length is expressed by

$\mathrm{Re}_{\mathrm{L}}=\mathrm{Re} . \mathrm{L}$

The dimensionless Stokes number of synthetic jet actuator fluidics is defined according to equation

$S=\sqrt{\frac{2 \pi f \mathrm{D}_{0}^{2}}{v}}$

The three key dimensionless parameters of synthetic jet in quiescent conditions namely, $L, S, \operatorname{Re}_{\mathrm{L}}$ related to each other via

$S=\sqrt{\frac{2 \operatorname{Re}_{L}}{L^{2}}}$

\section{BOUNDARY CONDITIONS COMPUTATIONAL MESH}

The computational domain of synthetic jet actuator as depicted in Figure 1 comprises of the vibrating diaphragm, the cylindrical cavity, the circular orifice, and the enclosing quiescent air inside which exits the synthetic jet and interacts with it. To ensure the similar relative displacements between different nodes of vibrating diaphragm and the incompressible fluid interior the cylindrical cavity, prismatic mesh is used. For stability of synthetic jet the hexahedral mesh is implemented for surrounding quiescent air and the circular orifice. The different types of computational meshes at different positions of synthetic jet actuator as depicted in Figure 2. The top wall, left wall and right wall of surrounding air is specified with pressure outlet boundary condition and the remaining walls are set as no slip boundary conditions having dimensions 80 orifice diameters along central axis and 100 orifice diameters along lateral direction to ensure that the synthetic jet at exit of the circular orifice is not influenced by applied pressure outlet boundary conditions. The no slip boundary condition is also specified for the cylindrical cavity and the orifice walls as seen in Figure 1.

\section{GRID AND TIME INDEPENDENCE STUDY}

The simulation time reduces by adopting the structured hexahedral mesh at outer ambient domain and unstructured prismatic grid at the cavity and the orifice. The moving mesh (ALE) technique of the CFD software is used for the oscillating diaphragm and cylinderical cavity to ensure the mesh velocity of diaphragm and fluid at interface are in the same phase. The medium coarse mesh with size of 120000 grid number is chosen for present study authenticated by grid independence test. Before computing the model for velocity and pressure field the solution is initialized with zero initial values of axial velocity, radial velocity and pressure field. A time step depends on actuation frequency is equal to $1 / 300 f$ is chosen for present study to allow for 3000 time steps per cycle. The convergence criteria in the residual monitors are set as $10^{-7}$.
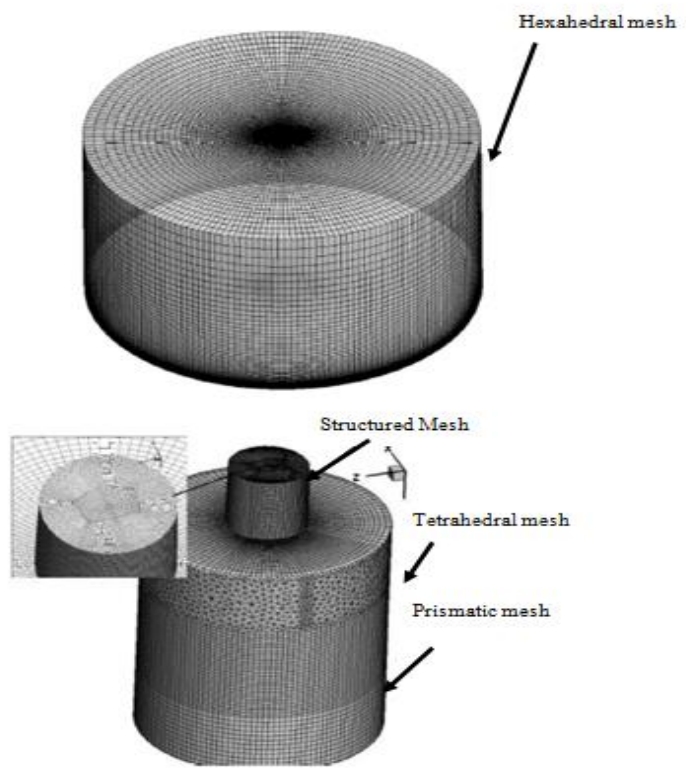

Figure 2. Three different types of meshes are utilized at different domains of the actuator at $f=10 \mathrm{~Hz}$

\section{VALIDATION OF NUMERICAL RESULTS}

For the purpose of validation of present computational study with that of existing experimental results of Mane et al. [13]. The cavity diameter $(\mathrm{Dc}=63.5 \mathrm{~mm})$, height of cavity $\left(\mathrm{H}_{\mathrm{c}}=5.5\right)$, orifice diameter $\left(\mathrm{D}_{\mathrm{o}}=2 \mathrm{~mm}\right)$ and height of orifice $\left(\mathrm{H}_{\mathrm{o}}=1.6 \mathrm{~mm}\right)$. The diaphragm displacement and operating frequency is chosen as $0.396 \mathrm{~mm}$ and $50 \mathrm{~Hz}$ respectively. Figure 3 depicts the comparison of radial velocity profile of present computational study and experimental result of Mane et al. [14] at a distance of $2 \mathrm{~mm}$ axially from the exit position of orifice. As seen from Figure 2 the radial velocity due to present computational study follows a similar trend but slightly different magnitudes. The percentage deviation between the present and the results revealed by Mane et al. [14] is $12.8 \%$.

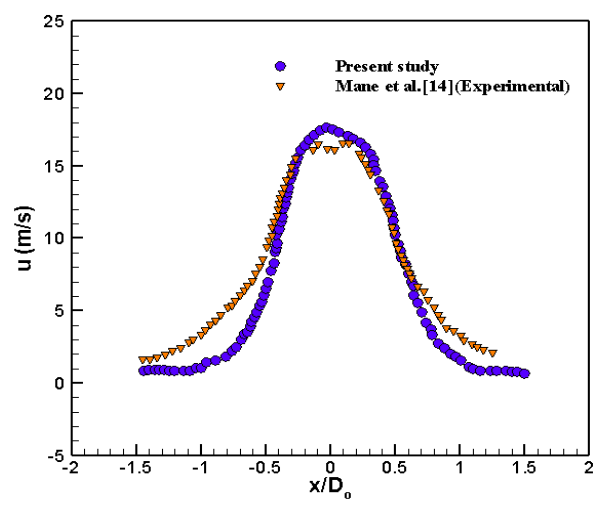

Figure 3. Comparison of radial velocity profile obtained from present numerical study ahead the existing experimental data of Mane et al. [14] at a distance of $2 \mathrm{~mm}$ from the exit orifice 


\section{RESULTS AND DISCUSSIONS}

Synthetic jets are characterized for the advection, evolution and dispersion of counter clockwise and clockwise vortex rings. The present study reports the characterization synthetic jet exit velocity at different dimensionless numbers

Figure 4(a-d) shows the vector velocity contour of synthetic jet actuator in quiescent flow in blowing phase at different times namely $\mathrm{T} / 8, \mathrm{~T} / 4,3 \mathrm{~T} / 8$ and $\mathrm{T}$ respectively operated at diaphragm displacement of $0.247 \mathrm{~mm}$ and actuation frequency of $8 \mathrm{~Hz}$. At the start of expulsion (corresponding to T/4) a vortex ring is formed due to shear layer at the orifice exit enhances rolls up of ambient air. As the fluid entrain into the cavity due to downwards movement of oscillating diaphragm (corresponding to $T / 4$ and $3 T / 4$ ) the vortex rings which is practically unaltered by the entrain of incompressible fluid in the cylindrical cavity grows independently and moves distant arising out of the orifice. As notice from Figure 4(d) (corresponding to 1T) the development of new vortex pair be subjected the evolution and promotion, peeling into the ambient fluid, encasing in the vacinity of the centre of the elementry vortices and fritter away to upstream.

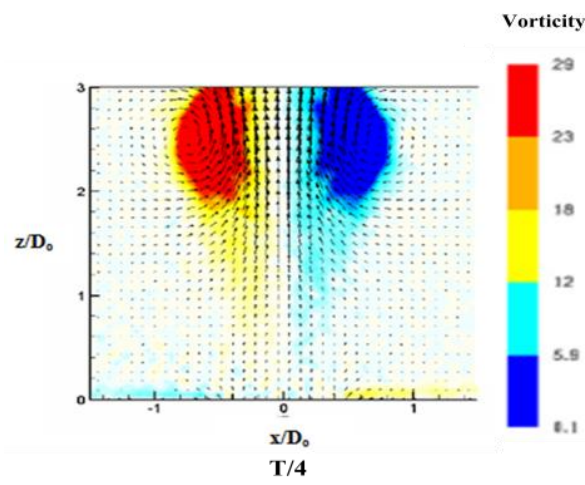

(a)

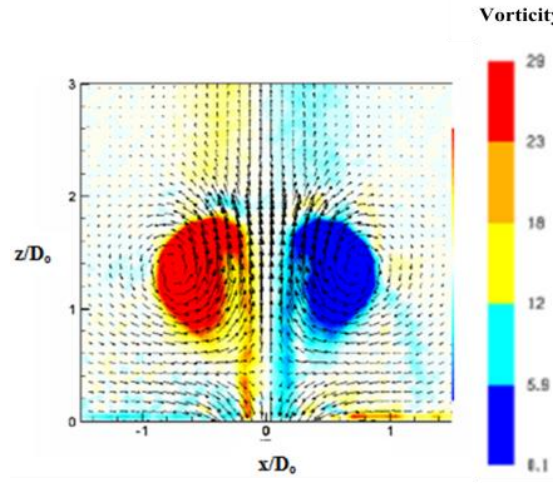

T/2

(b)

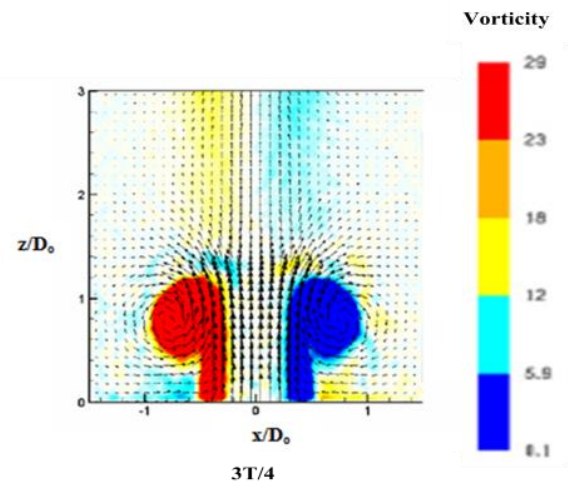

(c)

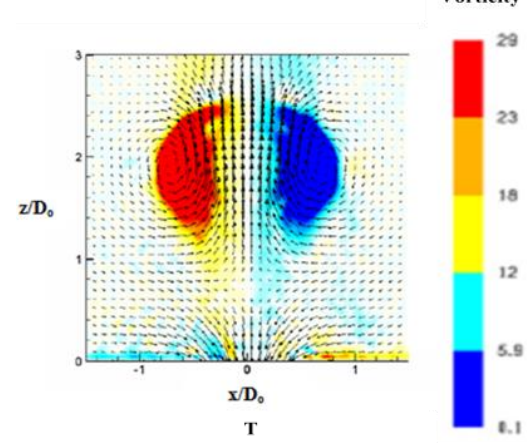

(d)

Figure 4. Surface velocity profile of Synthetic jet showing at velocity vectors at $f=10 \mathrm{~Hz}$ and $\Delta=0.247 \mathrm{~mm}$ and different times ( $\mathrm{t}=0$ to $\mathrm{T}$ )

The exit velocity of synthetic jet actuator is maximum at the centre of orifice. As we go away from orifice velocity decreases exponentially and becomes zero at the far away field due mixing of synthetic jet with the external quiescent flow field. The vortex rings appearing at the centre of orifice again justify maximum velocity at the centre.

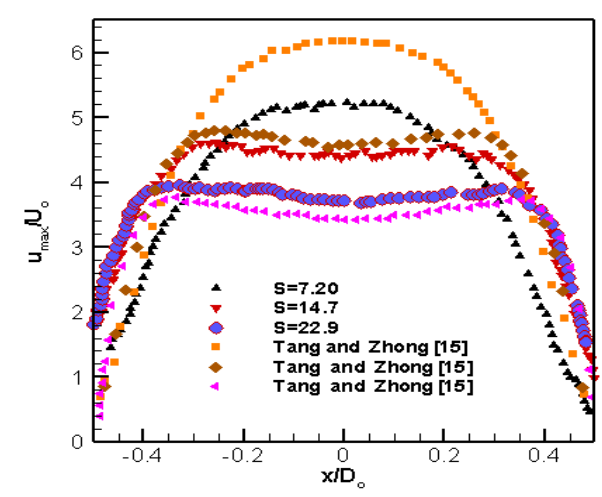

Figure 5. Comparison of exit velocity profiles at different Stokes Numbers at $\mathrm{L}=3$

Figure 5 shows exit velocity profiles at the constant stroke length $(\mathrm{L}=3)$ but different Stokes numbers and validated with experimental results of Tang and Zhong [15]. It is shown that velocity profile at the exit of orifice distorts evenly from close parabolic structure to a top-hat structure as (S) varies from 7.2 to 22.9. The percentage deviation between the present and the results revealed by Tang and Zhong [15] is $5 \%$.

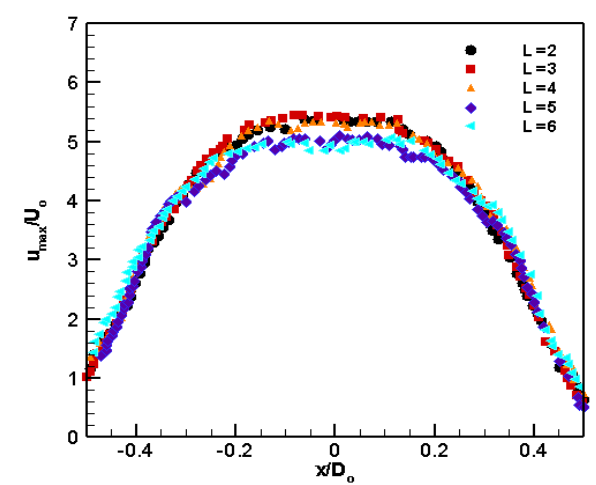

Figure 6. Comparison of exit velocity profiles at different dimensionless Strokes length at $\mathrm{S}=7.2$ 
Figure 6 shows exit velocity profiles at the same $(S=7.2)$ but different Strokes length. It is seen that exit velocity profile only changes slightly as the dimensionless stroke length increases from 2 to 6 which indicates that strokes length has little effect on exit velocity at small Stokes numbers. The effect of strokes length is more pronounced at higher stokes numbers especially at the central part of profile develops a plunge in the middle portion.

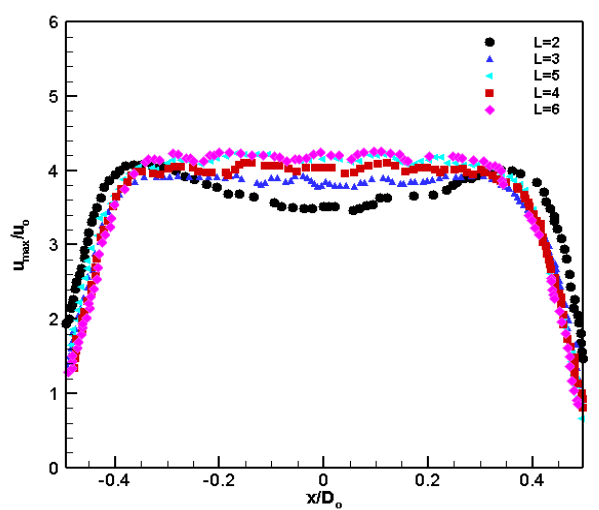

Figure 7. Comparison of exit velocity profiles at different dimensionless Strokes length at $\mathrm{S}=22.9$

Figure 7 shows the effect of Stokes number $(S=22.9)$ on the synthetic jet exit velocity profiles. It is seen that the effect of the dimensionless stroke length is more pronounced, particularly the middle portion of the exit velocity profile. At lower value of Strokes length $(\mathrm{L}=2)$ produced by the application of a lesser diaphragm displacement which leads a dip in the middle portion that stretches itself out by further increasing the dimensionless Stroke length.

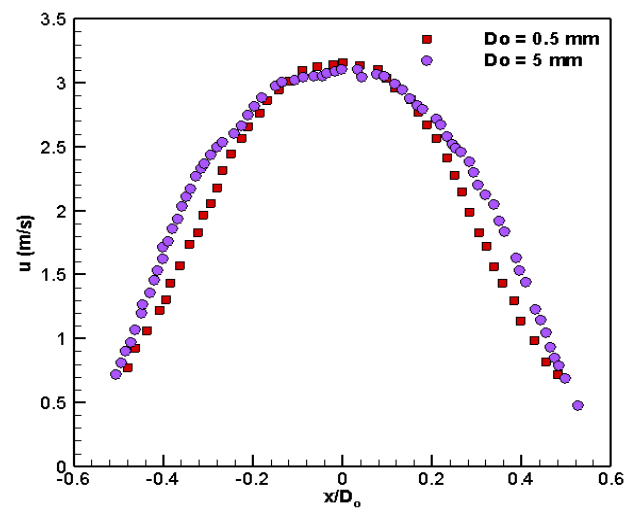

Figure 8. Comparison of jet exit velocity for macro and micro scale synthetic jet at $f=10 \mathrm{~Hz}, \mathrm{~S}=7.2$ and $\mathrm{L}=2$

Figure 8 predicts radial velocity profiles for two different orifice diameters. It is clear from Fig. 6 that velocity profiles have similar shapes because of slightly different values of $S$, $\mathrm{L}$ and $\mathrm{Re}_{\mathrm{L}}$. The weak roll-up is observed in the radial velocity profile of synthetic jet issued from $\mathrm{D}_{0}=0.5 \mathrm{~mm}$ as a result of low Stokes number and compressibility effect forming approximate parabolic exit velocity profile.

\section{CONCLUSIONS}

The detailed computational study on the characterization of dimensionless numbers such as Stroke lenth (L), Stokes number (S) and Reynolds number established on dimensionless Stroke length of synthetic jet actuator on fluid flow has been carried. The subsequent significant observations can be summerized as: The extend of vortex ring formation at the exit of orifice of the synthetic jet actuator depends upon the magnitude of Stroke length (L). At low Stroke length (L) the vortex rings formation severely weakens resulting the parabolic path of velocity profile. The strength and stability of vortex roll up determined by the Stokes number $(\mathrm{S})$ as it alters the structure of velocity profile by increasing the density of stokes layer in the interior of the orifice duct. For small orifice synthetic jet actuator operated at high frequency and exceeds the threshold value of Stokes number (S). The strength and stability of vortex roll up increase leads to effective flow control. This will provide the basic design code for synthetic jet flow parameters for optimum output.

\section{REFERENCES}

[1] Glezer A, Amitay M, Smith BL. (1998). Aerodynamics flow control using synthetic jet technology. Proceedings of $36^{\text {th }}$ AIAA Aerospace Sciences Meeting and Exhibit. Reno, NV, U.S.A. AIAA98-0208. https://doi.org/10.2514/6.1998-208

[2] Holman R, Utturkar Y, Mittal R, Smith BL, Cattafesta L. (2005). Formation criterion for synthetic jets. American Institute of Aeronautics and Astronautics Journal 43(10): 2110-2116. https://doi.org/10.2514/1.12033

[3] Jabbal M, Tang H, Zhong S. (2006). The effect of geometry on the performance of synthetic jet actuators. Proceedings of $25^{\text {th }}$ International Congress of the Aeronautical Science (ICAS). Hamburg, Germany. Paper ICAS 2006-P2.11.

[4] Smith BL, Swift GW, Kibens K. (2003). A comparison between synthetic jets and continuous jets. Experimental in Fluids 34(4): 467-472. https://doi.org/10.1007/s00348-002-0577-6

[5] Qayoum A, Gupta V, Panigrahi PK, Muralidhar K. (2010). Influence of amplitude and frequency modulation on flow created by a synthetic jet actuator. Sensors Actuators A: Physical 162(1): 36-50. https://doi.org/10.1016/j.sna.2010.05.008

[6] Zhang S, Zhong S. (2010). Experimental investigation of flow separation control using an array of synthetic jets. American Institute of Aeronautics and Astronautics Journal 48(3): 611-623. https://doi.org/10.2514/1.43673

[7] Watson M, Jaworski AJ, Wood NJ. (2003). Contribution to the understanding of flow interactions between multiple synthetic jets. American Institute of Aeronautics and Astronautics Journal 41(4): 747-749. https://doi.org/10.2514/2.2008

[8] McGuinn A, Farrelly R, Persoons T, Murray DB. (2013). Flow regime characterization of an impinging axisymmetric synthetic jet. Experimental Thermal Fluid Science 47: 241-251. https://doi.org/10.1016/j.expthermflusci.2013.02.003

[9] Glezer A, Amitay M. (2002). Synthetic jets. Annual Review of Fluid Mechanics 34: 503-529. https://doi.org/10.1146/annurev.fluid.34.090501.094913

[10] You D, Moin P. (2007). Study of flow separation over 
an airfoil with synthetic jet control using Large-eddy simulation. Center for Turbulence Research-Annual Research Briefs 45: 311-321.

[11] Jain M, Puranik B, Agrawal A. (2011). A numerical investigation of effects of cavity and orifice parameters on the characteristics of a synthetic jet flow. Sensor and Actuators A: Physical 165(2): 351-366. https://doi.org/10.1016/j.sna.2010.11.001

[12] Lv Y, Zhang J, Shan Y. (2014). Numerical investigation for effects of actuator parameters and excitation frequencies on synthetic jet fluidic characteristics. Sensor and Actuators A: Physical 219: 100-111. https://doi.org/10.1016/j.sna.2014.08.009

[13] Batikh A, Caen R, Colin S, Kourta A, Boisson H. (2008). Numerical and experimental study of micro synthetic jets for active flow control. International Journal of Heat and Technology 26(1): 139-145. https://doi.org/10.18280/ijht.260119

[14] Mane P, Mossi K, Rostami A, Brayant R, Castro N. (2007). Piezoelectric actuators as synthetic jet cavity dimension effects. Journal of Intelligent Material System and Structure 18: 1175-1190. https://doi.org/10.1177/1045389X06075658

[15] Tang H, Zhong S. (2005). 2D numerical study of circular synthetic jets in quiescent flows. The Aeronautical Journal 109(1092): 89-97. https://doi.org/10.1017/S0001924000000592

\section{NOMENCLATURE}

$\mathrm{D}_{\mathrm{c}} \quad$ cavity width, $\mathrm{m}$

$\mathrm{D}_{\mathrm{o}} \quad$ orifice width, $\mathrm{m}$

$f \quad$ actuation frequency of diaphragm, $\mathrm{m}$ $\mathrm{h}_{\mathrm{c}} \quad$ height of cavity, $\mathrm{m}$

$h_{0} \quad$ height of orifice, $m$

L Stroke length, dimensionless

p pressure, pa

Re Reynolds number, dimensionless

$\mathrm{Re}_{\mathrm{L}} \quad$ Reynolds number established on dimensionless Stroke length, dimensionless

$\begin{array}{ll}\mathrm{S} & \text { Stokes number, dimensionless } \\ \mathrm{S}_{\mathrm{r}} & \text { Strouhal number, dimensionless }\end{array}$

$\mathrm{T} \quad$ time period, $1 / \mathrm{s}$

t time, $\mathrm{s}$

$\mathrm{u} \quad$ radial velocity field, $\mathrm{m} / \mathrm{s}$

$\mathrm{U}_{\mathrm{o}} \quad$ average velocity of synthetic jet, $\mathrm{m} / \mathrm{s}$

$\mathrm{u}_{\mathrm{t}} \quad$ instantaneous velocity of synthetic jet, $\mathrm{m} / \mathrm{s}$

$\mathrm{u}_{\max } \quad$ peak velocity at orifice exit, $\mathrm{m} / \mathrm{s}$

B1 axial dimensions of quiescient air, $\mathrm{mm}$

B2 lateral dimensions of quiescient air, $\mathrm{mm}$

\section{Greek symbols}

$\rho \quad$ density of fluid, $\mathrm{kg} / \mathrm{m}^{3}$

$\mu_{\mathrm{T}} \quad$ kinematic turbulent viscosty, $\mathrm{m}^{2} / \mathrm{s}$

$\mu \quad$ dynamic viscosity, $\mathrm{kg} / \mathrm{ms}$

$v \quad$ kinematic viscosity, $\mathrm{m}^{2} / \mathrm{s}$

$\Delta \quad$ diaphragm displacement, $\mathrm{mm}$

\section{Subscript}

$\begin{array}{ll}\text { o } & \text { average } \\ \mathrm{cl} & \text { centreline } \\ \mathrm{t} & \text { instantaneous } \\ \text { max } & \text { maximum } \\ \mathrm{i} & \text { inlet } \\ \mathrm{w} & \text { wall }\end{array}$

